\title{
DESEMPEÑO ACADÉMICO EN LAS PRUEBAS SABER 11
}

Gustavo Adolfo Junca Rodríguez Universidad Nacional de Colombia Colombia 


\title{
Desempeño academico en las pruebas Saber 11 Resumen
}

En este artículo se analiza el desempeño en matemáticas de los estudiantes de bachillerato en las pruebas realizadas para ingreso a al educación superior (Pruebas Saber 11) en el caso de Bogotá. La enseñanza y el aprendizaje de las matemáticas conlleva el aprendizaje de un conjunto de objetos matemáticos, así como la representación, además del aprendizaje de un conjunto de operaciones y relaciones que modifican los registros de representación semióticos en el mundo matemático. En el caso de las Pruebas Saber 11 se busca mirar el desempeño en tres conjuntos de competencias: Argumentativa, Comunicativa y de Resolución de Problemas. El trabajo analiza a través de un modelo multinivel jerárquico lineal (HGLM) el impacto en el desempeño heterogéneo de los estudiantes dependiendo, en el primer nivel de factores Individual y Capital Social (familia); y en el segundo nivel el factor de entorno escolar. Uno de los resultados importantes es mostrar que el desempeño depende de los resultados en lenguaje. De igual manera, en términos del cambio de representación, el desempeño depende de que el estudiante tenga acceso a computador en la casa. Finalmente, los resultados muestran un efecto de género donde los resultados individuales favorecen a los hombres, mientras que los resultados en conjunto favorecen a las mujeres por colegio.

Palabras clave: Desempeño académico; habilidades; multinivel

\section{Performance academique dans les tests Sabre 11}

\section{Résumé}

Cet article étudie un score de performance en mathématiques pour examen gouvernemental (Pruebas Sabre 11) pour les élèves des écoles afin d'accéder à un troisième cycle universitaire ou technologique à Bogotá (Colombie). Les processus d'enseignement et d'apprentissage en mathématiques réalisent l'apprentissage des objets mathématiques et de leurs représentations, mais aussi le processus d'apprentissage d'un ensemble d'opérations et de relations entre ces objets, qui a également modifié ou créé un ensemble de registres sémiotiques. Pour Pruebas Sabre 11, nous avons analysé les compétences de performance de l'élève sur: Tâche d'argumentation, de communication et de résolution de problèmes. Nous estimons un modèle à plusieurs niveaux en deux étapes ou niveaux. Le premier prend en compte les facteurs de capital individuel et social, tandis que le second inclut les caractéristiques de l'école. Un résultat important est que nous trouvons se réfère à l'impact des performances linguistiques dans les performances mathématiques. De la même manière, la performance individuelle en mathématiques est corrélée positivement avec l'élève ayant accès à l'ordinateur à la maison. Enfin, nous trouvons un résultat actuel où les jeunes hommes ont de meilleurs résultats que les jeunes femmes au niveau individuel, mais en tant que groupe, les femmes ont de meilleurs résultats que les hommes.

Mots-clés: Performance académique; compétences; modèle à plusieurs niveaux

\section{Academic performance in the Saber 11 tests}

\begin{abstract}
This paper study a performance score in mathematics for government examination (Pruebas Saber 11) for school students in order to get access to undergraduate or technological third education level in Bogotá (Colombia). Teaching and learning processes in Mathematics realize learning of mathematics objects and its representations, but also learning process of a set of operations and relations among these objects, that also modified or create a set of semiotics registers. For Pruebas Saber 11 we analyzed the student's performance competencies on: Argumentative, Communicative and Problem Resolution task. We estimate a multilevel model in two stages or levels. The first one takes in account individual and social capital factors, while the second includes school characteristics. An important result is that we find refers the impact of language performance in math performance. In the same way, individual performance in mathematics is positive correlated with the student has access to computer at home. Finally, we find a current result where young men have better results than young women at individual level, but as a group, women have better results than men.
\end{abstract}

Keywords: Academic performance; skills; multilevel 


\section{Desempeño academico en las pruebas Saber 11}

\section{INFORMACIÓN DEL ARTíCULO}

Recepción del artículo: 05/06/2018

Concepto de evaluación: 18/07/2018

Aceptación del artículo: 03/08/2018
Gustavo Adolfo Junca Rodríguez* Universidad Nacional de Colombia, Colombia

\section{INTRODUCCIÓN}

El aprendizaje de los Objetos Matemáticos y el Lenguaje son esenciales para el aprendizaje y el desarrollo de conocimientos especializados en las ciencias naturales y sociales; de allí la necesidad de realizar evaluaciones que midan el desempeño en estas dos áreas durante la educación primaria, básica y secundaria. En Colombia, durante los últimos 30 años, el Instituto Colombiano para el Fomento de la Educación Superior (ICFES) ha aplicado Exámenes de Estado (antes examen ICFES ahora Prueba Saber11) a los estudiantes que terminan su educación secundaria y desean acceder a la educación superior universitaria ${ }^{1}$. Es así como el peso de los componentes de matemáticas y lenguaje dentro del puntaje ponderado por estudiante es tres veces mayor ${ }^{2}$.

En la última década, la existencia de los Exámenes de Estado y el acceso a las bases de datos sobre el desempeño de los estudiantes en los distintos componentes ha permitido un conjunto de estudios con miras a explicar el desempeño de los estudiantes y este aspecto se ha ido consolidando como un campo de estudio en sí mismo. Los distintos trabajos

*Profesor Asociado de la Universidad Nacional de Colombia. Grupo de Investigación Interdiscplinario en Pedagogía del Lenguaje y las Matemáticas - Doctorado Interinstitucional en Educación. Cualquier error es responsabilidad del autor. Autor de correspondencia: gajuncar@unal.edu.co

1. Durante este tiempo la prueba tuvo dos grandes modificaciones. La primera en el año 2000 con una reducción del número de preguntas y una mayor dificultad. En ese año se comenzó a medir el desempeño de los colegios. En la actualidad, la prueba se rige por la Resolución No. 489 del 20 de octubre de 2008 donde se especifica la forma de medición del desempeño del estudiante y el de los planteles.

2. El promedio ponderado se calcula, según la Resolución No. 489 del 20 de octubre de 2008, sumando el puntaje obtenido en cada una de las áreas de Química, Biología, Física, Filosofía e Inglés. Luego se suma el doble del puntaje obtenido en el área de Ciencias Sociales, y finalmente se suma el triple del puntaje obtenido en las áreas de Matemáticas y Lenguaje. 
enfatizan aspectos socieconómicos y efectos plantel (colegios públicos o privados), entre otros. De igual manera la mayoría de los estudios buscan explicar el desempeño en los resultados globales de las prueba, así como de cada uno de sus componentes y las relaciones entre ellos, en función de otros factores de carácter principalmente socieconómico.

Como menciona Rodríguez (2011), el debate sobre el reforzamiento o no de los procesos de estratificación y segregación social continúa abierto; sin embargo, la educación en Colombia muestra una persistente desigualdad educativa a nivel nacional y en particular a nivel de Bogotá. Los distintos trabajos para Colombia muestran que el principal factor que explica el desempeño, controlando por efecto plantel, rural/urbano, estrato, es el contexto familiar, en particular las características socieconómicas parecen ser el factor más importante como determinante del logro educativo medido por los resultados en las pruebas Saber11 (Barrera (2005), Barrientos y Gaviria (2001a), Barrientos y Gaviria (2001b), Barrientos y Gaviria (2001c), Becerra, González y Sarmiento (2000)).

El presente estudio busca analizar el desempeño en la prueba de matemáticas a nivel de pregunta y por competencias a través de un modelo multinivel (HGLM: Hierarchical Generalized Linear Model). Para el análisis por niveles se construyeron 14 grupos, combinando la clasificación de Calidad del ICFES y la naturaleza del plantel (público o privado) ${ }^{3}$. En particular, el estudio busca arrojar luces sobre tres conjuntos de características con relación a: el individuo, el capital social y el entorno escolar, que afectan el desempeño académico. El estudio muestra que los principales determinantes que afectan el desempeño de los estudiantes en el componente de matemáticas, para las competencias comunicativa, argumentativa y de solución de problemas, son las características del individuo. Para los aspectos relacionados con el entorno familiar y el entorno escolar los resultados varían entre Bogotá y el Nivel Nacional. De igual manera, el estudio muestra que los aspectos socieconómicos, medidos a través del ingreso del hogar y el índice socieconómico del colegio, son relevantes, como muestran los distintos estudios mencionados anteriormente.

El documento consta de siete partes, de las cuales la presente introducción es la primera. La segunda parte presenta los fundamentos teóricos para abordar el análisis del desempeño de los estudiantes en las pruebas de matemáticas. En la tercera sección se especifica el HGLM en dos niveles. La cuarta y quinta partes presentan la estimación del modelo y los resultados. La última plantea las conclusiones.

\section{ENSEÑANZA Y APRENDIZAJE DE LAS MATEMÁTICAS}

De acuerdo con Godino y col. (2006), existen tres enfoques teóricos en didáctica de las matemáticas ${ }^{4}$ :

- Programa epistemológico de investigación en didáctica de las matemáticas, que toma como base del análisis un modelo de estructura y dinámica de la actividad

\footnotetext{
3. Los trabajos multinivel, para Colombia, analizan el desempeño con base en el puntaje global obtenido en la prueba (Rodríguez (2011), Correa (2004)).

4. Como menciona D’Amore (2006) el término didáctica hace referencia no solo la divulgación de las ideas o la fase de la enseñanza (Didáctica A), sino también fijando la atención en la fase del aprendizaje o epistemología del aprendizaje de las matemáticas (Didáctica B).
} 
escolar (Bosh, Fonseca y Gascón (2004)) en el cual están ubicadas la teoría de situaciones (Brousseau (1986), Brousseau (1998)), la dialéctica instrumento-objeto (Douady (1986), Lerouge (2000)) y la teoría antropológica (Chevallard (1999)).

- Programa cognitivo en didáctica de las matemáticas que comprende la teoría de los campos conceptuales Vergnaud (1990), la noción de concepción Artigue (1990) y los registros de representación semiótica Duval (2004).

- Programa onto-semiótico o del Enfoque Onto-Semiótico (EOS) busca integrar elementos de los dos enfoques anteriores. (Godino y Batanero (1994), Godino (2002), Godino, Batanero y Roa (2005), Godino, Contreras y Font (2006)).

Dentro de estas perspectivas de investigación haremos énfasis en algunos aspectos que muestran la complejidad y dinámica de los procesos de enseñanza y aprendizaje de las matemáticas, lo que conlleva a un análisis sistémico de dicho proceso. La dimensión compleja de los procesos de enseñanza y aprendizaje se ve reflejada en las diversas categorías abordadas en cada uno de los tres programas mencionados para abordar los distintos enfoques teóricos en didáctica de las matemáticas.

El aspecto dinámico refleja no sólo el proceso de acumulación de conocimiento de los objetos matemáticos durante la educación primaria, básica y media, sino que dicho proceso está marcado por problemas de conceptos errados o misconcepciones; errores que persisten en el tiempo y que son fruto de ciertos aprendizajes desarrollados en etapas tempranas del aprendizaje de las matemáticas. Este tipo de misconcepciones originados por errores conceptuales de los maestros, o que emergieron en el proceso de enseñanza pero que no fueron detectados por el maestro, persisten y pueden afectar el desempeño de los estudiantes en pruebas estandarizadas. Dichas misconcepciones se pueden reflejan en una baja tasa de respuesta de un ítem en particular o en el elevado número de respuestas incorrectas. En efecto, este bajo desempeño en las áreas de matemáticas por parte de los estudiantes colombianos, ya sea en pruebas nacionales como la de saber11, ya en los resultados de pruebas internacionales como es el caso de la prueba Pisa, muestra las dificultades de los estudiantes en los procesos de enseñanza y aprendizaje de las matemáticas.

La Figura 1 muestra la competencia de solución de problemas evaluada en las Pruebas Saber 11 en el 2009. Es claro que la distribución de la respuesta al ítem no es simétrica. En promedio los estudiantes de Bogotá D.C. responden el 31\% de las preguntas que evalúan la competencia de solución de problemas, mientras que a nivel nacional el porcentaje es del $27 \%^{5}$. Para las otras competencias el porcentaje de respuesta es mayor pero muy inferior al $50 \%$. 
Figura 1. Distribución de respuesta al ítem. Competencia Solución de Problemas

Bogotá D.C.
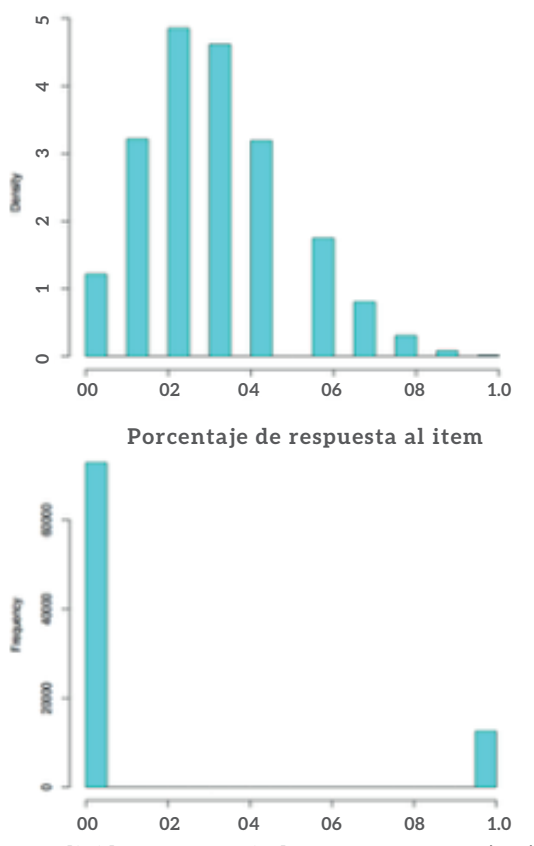

Total Nacional
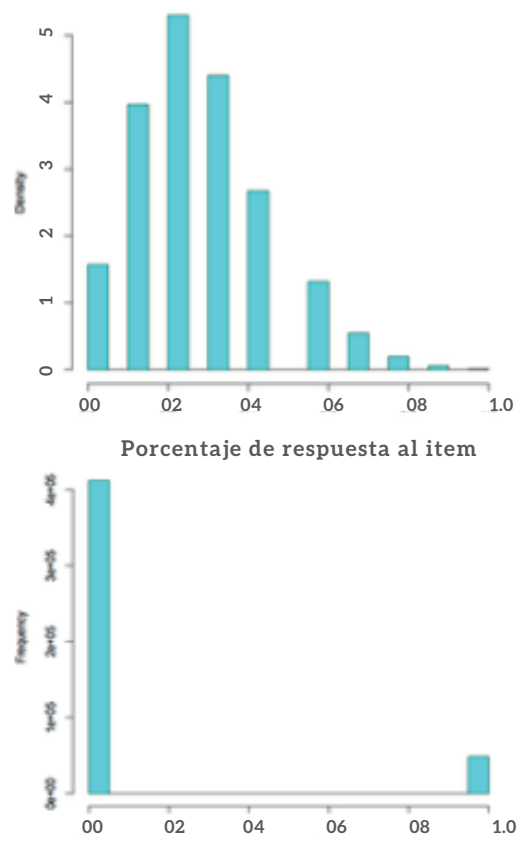

Individuos porcentaje de respuesta: $0=\operatorname{Porc}\left({ }^{0} .5\right)$

\section{Fuente ICFES. Cálculos del Autor}

De igual manera, como plantea Duval (2004), como los objetos matemáticos no son ostensibles, el proceso de aprendizaje de las matemáticas está afectado por la representación semiótica de los objetos matemáticos. En primer lugar, la decisión de un tipo de Registro de Representación Semiótica (RRS) (geométrica, diagrama cartesiano, una problema matemático enunciado en lengua natural, o una combinación de diversos tipos de RRS) buscará enfatizar distintos elementos de un mismo objeto matemático. Una vez se ha escogido el registro(s) de representación semiótica, el individuo que aprende se enfrenta a las cambios o transformaciones de dicha representación. El primero tipo de cambio es el tratamiento, es decir, una transformación de una representación a otra dentro del mismo registro semiótico, mientras que la conversión hace referencia a los cambios entre distintos tipos de representación de un RRS a otra representación de otro RRS.

Como afirma Duval (2004), los procesos de aprendizaje son posibles por las distintas representaciones semióticas; es decir, no hay noesis (acto de pensar, conocer y entender un objeto matemático) sin semiosis (representación del objeto matemático). De manera que el acto de entender un objeto matemático se refleja en la capacidad para discurrir entre distintos tipos de representación semiótica. Una prueba estandarizada como la prueba Saber11 o la prueba Pisa busca movilizar un conjunto de conocimientos y evaluar las capacidades de los estudiantes para responder un problema estrictamente sobre objetos 
matemáticos o sobre el uso de estos objetos en problemas asociados a otras áreas del conocimiento en donde el conocimiento de los objetos matemáticos es esencial. Y este conocimiento (noesis) implica el manejo de los distintos tipos de registro de representación semiótico (semiosis).

\section{LA TEORÍA GENERAL DE SISTEMAS Y LOS PROCESOS DE ENSEÑANZA Y APRENDIZAJE DE LAS MATEMÁTICAS}

Olsson y Sjöstedt (2005) entienden la Teoría General de Sistemas (TGS) como un modo de pensamiento acerca de los problemas complejos. En general, un sistema es una interacción entre un conjunto de elementos sistémicos separados de un ambiente externo. El sistema está relacionado con sus ambiente a través de un conjunto de insumos (inputs) y un conjunto de resultados o productos (outputs). Vasco (1995) describe un sistema como un conjunto de elementos que interactúan entre ellos, sufriendo ciertas transformaciones y sujetos a ciertas relaciones. Vasco (1995) entiende la TGS como una metodología estática y simplificada, para analizar y entender los más diversos procesos dinámicos y complejos ${ }^{6}$.

El científico ruso Bogdanov (1873-1928) es reconocido como el precursor de la TGS y de la Cibernética, pero el trabajo más influyente sobre la TGS es debido a Bertalanffy (1968) donde compila sus artículos científicos del periodo 1940-1968. Los otros trabajos seminales que permitieron el desarrollo de la TGS son los de Norbert Wiener y Ross Ashby sobre cibernética.

Con miras a aplicar la Teoría General de Procesos y Sistemas (TGPS) Vasco (1995) desde su perspectiva metodológica, nosotros definimos el proceso como la unidad de análisis, como el objeto de estudio. El proceso es un fenómeno que tiene dos caracterís- ticas. La primera es que es dinámico (cambiante) en el tiempo y el espacio. La segunda es que es complejo o múltiple. Un proceso a su vez puede estar compuesto por otros subprocesos o ser parte de un proceso más general. Por ejemplo el proceso educativo ${ }^{7}$ podría decirse que está conformado por los procesos de enseñanza y de aprendizaje, mientras que el proceso educativo podría entenderse como parte de un proceso más amplio como el de formación.

Una vez definido el proceso a estudiar, entonces procedemos a definir el conjunto de elementos externos (agentes/actores/insumos) e internos (componentes) que deberían ser considerados como parte relevante del proceso; aquellos elementos o factores deberían ser vistos como pertenecientes a su ambiente, y éstos como pertenecientes al sistema; con el fin de "limitar" el problema a estudiar.

\footnotetext{
6. Nosotros evitamos la discusión teórica acerca del "sistema" como el objeto de estudio de la TGS, para esta discusión ontológica ver Luhmann (1998) y Lilienfeld (1984). Siguiendo a Vasco (1995), nosotros entendemos lo real como un proceso en si mismo, es decir un fenómeno dinámico (en el tiempo y el espacio) y complejo. Mientras que la Teoría de Sistemas es una manera de pensar y analizar cualquier proceso. Olsson y Sjöstedt (2005) y Ulrich (2001) ven la Teoría de Sistemas, desde la misma perspectiva, como una metodología.

7. La Educación Formal la entendemos comola organización de un conjunto de conocimientos, in- fraestructura e individuos (profesores y estudiantes) con miras a la obtención de un título o grado educativo: Educación Básica, Educación Secundaria, Educación Técnica, Educación Tecnológica, Edu- cación Universitaria de Pregrado y Posgrado.
} 
La Figura 2 muestra una representación estándar de un sistema. Los insumos (inputs) del proceso representan los elementos externos relevantes que interactúan en el proceso con miras a producir un resultado o producto (output). El sistema, entendido como la interacción de los componentes con los insumos, puede ser o no caracterizado a través de un modelo explícito, o como en muchos casos, dependiendo de nuestro grado de comprensión, se representaría con una "caja negra". En el caso del proceso educativo, el proceso de aprendizaje implica que los individuos reciben un conjunto de información a través de una conferencia, una clase magistral, la lectura de un libro, etc. Dicha información puede estar representada en estructuras multi-registro que el individuo a través del proceso cognitivo incorpora a su conjunto de conocimientos, sean estos teóricos o prácticos. Todas estas representaciones provienen del ambiente externo y las consideramos como insumos (inputs).

Si bien aún no tenemos total certeza del proceso cerebral interno (bio-químico) que acompaña el acto de conocer, el grado en el cual dicho conocimiento es incorporado puede ser evaluado o medido de manera indirecta ${ }^{8}$. En la teoría de la medición de pruebas escritas se considera que el sistema nervioso central (SNC) es una caja negra de la cual no tenemos información directa. Es posible que algún día los TAC, PET, electro y magnetoencefalograma u otros artefactos todavía por inventar permitan evaluar en tiempo real el estado de conocimiento lingüístico o matemático del SNC de un sujeto individual. Pero en la aplicación de pruebas escritas sólo podemos medir indirectamente ese estado de conocimiento a través de la presentación de una representación semiótica mono-o multiregistro, solicitando al sujeto que la transforme en otras que él mismo pueda producir mentalmente y externalizar (pregunta abierta) o al menos que le permitan seleccionar cuál de las predeterminadas considera más cercana a la que él produjo mentalmente según las instrucciones dadas (pregunta cerrada).

No es parte del problema averiguar que tan bien desagregado está el ambiente en aquellos factores que denominamos "asociados" al desempeño del sistema objeto de estudio, ni qué tan bien medidas están las variaciones en esos factores asociados a los valores codificados en la base de datos. Tenemos que aceptarlos como dados y dados así ("data").

Esos factores asociados influyen en cada uno de los sistemas individuales que llamamos "estudiante de grado 110", y por lo tanto no podemos asociarlos directamente con el comportamiento emergente de un posible sistema llamado "curso de grado 110" de un colegio X, y mucho menos con otro sistema llamado "colegio X".

El problema de estudio está delimitado por los elementos del ambiente que afectan al sistema y por los que éste emite como respuesta (inputs-outputs) y las interacciones entre ellos. El ambiente externo puede ser representado por un proceso más general o desagregado en factores exógenos que pueden o no afectar el sistema (representación estática del proceso). Es así como el sistema, seleccionado como objeto de estudio, así como la interacción de sus elementos, es un constructo mental o un modelo que nos permite entender un proceso particular. 
Otro aspecto central hace referencia al análisis cualitativo de su comportamiento, que depende de la interacción de los elementos. Este aspecto es conocido como el comportamiento emergente del sistema. Por este comportamiento emergente se le atribuye a un sistema la "racionalidad", el "propósito" o el "objetivo" del sistema. Ese comportamiento emergente con el propósito que se le atribuye es parte del constructo mental o el modelo hecho por el observador (investigador). En la TGS, este "propósito" supone que los sis- temas por si mismos tienen un fin teleológico, en especial en los sistemas biológicos, que buscan una autorregulación para lograr una finalidad, que a veces llamamos "conservar la vida" o "preservar la especie".

El concepto de retroalimentación o feedback es tomado de la teoría de la información. En algunos casos, hace referencia al impacto sobre el resultado final de estímulos externos en un simple esquema input-output cuando esos estímulos externos se han modificado por el "output" previo del mismo sistema. En otras situaciones, el mecanismo de retro alimentación podría ser visto como un sistema complejo en si mismo que permite, como en el caso de los organismos, el equilibrio o balance (homeostático) de ciertas funciones como la regulación de la temperatura interna del organismo, función necesaria para la vida. En cualquier caso, tener en cuenta el feedback exigiría estudios longitudinales y análisis diacrónicos con series de tiempo que están por fuera del alcance del presente estudio.

Finalmente otra distinción es entre un sistema abierto o cerrado. La distinción hace referencia a la manera como se considera la frontera entre el sistema y su ambiente. $\mathrm{Si}$ el sistema es cerrado, las interacciones entre los elementos internos del sistema es lo que importa. De igual manera, en un sistema cerrado, el estado final depende de las condiciones iniciales internas o estado inicial, mientras que en los sistemas abiertos, es posible que a partir de distintas condiciones iniciales internas se llegue al mismo estado final, o a partir del mismo estado inicial se llegue a distintos estados finales.

Aunque ciertos modelos, llamados "autopoyéticos" de los sistemas vivos intentan representar a cada estudiante como un sistema informáticamente cerrado, consideramos que cada uno de los sujetos de este estudio se representa en forma más potente como sistema triplemente abierto, en el sentido de permeabilidad de fronteras para el intercambio de materia, de energía y de información. De lo contrario, no podríamos atribuirle influencia alguna en el comportamiento en las pruebas a la buena o mala alimentación, a las condiciones confortables o no del vestido, la vivienda y el aula de clase, o a la presencia o no de libros y computadores en casa.

El momento en que se toman las pruebas estandarizadas podría verse como un corte en el tiempo del proceso de aprendizaje de un estudiante para evaluar un conjunto de conocimientos. Una vez practicada la prueba y obtenidos los resultados, nos interesa analizar el desempeño de cada sujeto y cuáles factores están asociados a un mejor desempeño en la prueba. 


\section{EL DESEMPEÑO EN LAS PRUEBAS SABER11 DESDE UN ENFOQUE DE TEORÍA GENERAL DE PROCESOS}

La prueba Saber11, así como cualquier prueba estandarizada en los distintos campos de conocimiento, evalúa si el conocimiento personal (la apropiación individual del campo conceptual, institucionalizado con sus nociones, conceptos y concepciones) que emerge de las situaciones didácticas en el aula (teoría de las situaciones) y que conforma el saber personal, en este caso, el saber matemático del individuo, se corresponde con Saber Sabio o conocimiento institucional aceptado en una disciplina, en este caso, las matemáticas en sí mismas y en relación con otras ciencias y con la vida diaria. Desde la perspectiva del ICFES:

"En las pruebas se asumirá la perspectiva integradora de los Lineamientos
y Estándares, respecto a los conocimientos básicos, procesos y contextos,
privilegiando como contexto las situaciones problemáticas enmarcadas en las
mismas matemáticas, la vida diaria y las otras ciencias." (Acevedo y col. (2007),
p. 22)

De manera que emerge además la pregunta por aquellos aspectos que pueden afectar el desempeño, es decir, ¿cuáles factores individuales, socio-culturales y relacionados con el entorno escolar pueden afectar el desempeño del individuo? Tanto la teoría de las situaciones propuesta por Brousseau (1986) y los trabajos de Bronfenbrenner (1979) sobre la teoría ecológica de sistemas, plantean el medio como elemento central dentro de los procesos de enseñanza y de aprendizaje.

Como plantea D’Amore (2006) para Brousseau el fenómeno de la enseñanza y el aprendizaje es un sistema didáctico y, como sistema conformado por tres elementos: maestro, estudiante, y saber; inmersos en un medio externo "naturalmente existe un mundo ex- terno, la sociedad en general, los padres, los matemáticos, etcétera" que forman parte del sistema y lo afectan. El concepto de medio ("milieu") es un concepto complejo que lleva consigo elementos explícitos e implícitos que están presentes y afectan de manera directa e indirecta las situaciones didácticas en la escuela.

Para Bronfenbrenner (1979) existen diversos niveles de interacción que influyen en el desarrollo del individuo, como son las interacciones entre sus pares, la familia, la escuela, y la comunidad.

El problema es si estos factores se consideran dentro o fuera de las fronteras del sistema objeto de estudio (lo que revela la relativa arbitrariedad de la ubicación de las fronteras del sistema). En nuestro caso, queremos hacer énfasis en las características individuales, el capital social de la familia y el entorno escolar como ámbitos que afectan el desempeño académico en general y el desempeño en matemáticas en particular. El entorno comunitario sólo se tendría en cuenta si se hicieran análisis con variables como rural/urbano, o comparaciones por ciudades o regiones de Colombia, las cuales no analizamos aquí. 


\subsection{El enunciado matemático}

En la frontera entre el estudiante como sistema objeto de estudio y su ambiente en el momento de corte sincrónico del proceso de educación básica y media que señalamos como la toma de la prueba Saber 11, el input es el enunciado de cada pregunta.

Una pregunta o ítem busca evaluar un concepto o conjunto de conceptos que tiene el individuo sobre uno o varios objetos matemáticos que se relacionan entre si y que son entendidos de manera específica y particular por una comunidad matemática asumida como universal, independiente de si la comunidad local, la escuela, o la familia los entiende así o no. Con los datos que se tienen no es posible comprobar que al estudiante en cuestión "le hayan enseñado mal" un concepto o que haya en error en su libro de texto escolar. Si un individuo responde de manera correcta o incorrecta, o aun si se abstiene de responder una pregunta particular; esto refleja una ruptura entre lo que podríamos llamar el saber institucional o "saber sabio" y el saber individual o personal.

"La evaluación de la competencia matemática está referida al saber hacer en el contexto matemático escolar, [nota] decir, a las formas de proceder aso- ciadas al uso de los conceptos y estructuras matemáticas. La aproximación que se hace a la competencia matemática en la prueba tiene en cuenta las significaciones que el estudiante ha logrado construir y que pone en evidencia cuando se enfrenta a diferentes situaciones problema. En las pruebas es importante evaluar el significado de los conceptos matemáticos y la práctica significativa, relacionada esta última con la matematización que exige al estudiante simbolizar, formular, cuantificar, validar, esquematizar, representar, generalizar, entre otros, [a] ctividades [que] le permitirán desarrollar descripciones matemáticas, explicaciones o construcciones." (Acevedo y col. (2007), p. 22)

Si bien no tenemos acceso directo a las preguntas formuladas en el componente de matemáticas, de la información suministrada sabemos la competencia ${ }^{9}$ que busca evaluar la pregunta y, en segundo lugar, el componente ${ }^{10}$, es decir, los aspectos conceptuales y estructurales al que hace referencia la pregunta. En el caso de las pruebas Saber11 para el área de matemáticas, la prueba está estructurada para evaluar tres competencias: comunicación, razonamiento y solución de problemas; en tres tipos de componentes: Numérico-variacional, Geométrico-métrico y Aleatorio. Las competencias que evalúa la prueba de matemáticas aparecen descritas en la Tabla 1.

\footnotetext{
9. Existen cinco procesos generales que tienen que ver con el aprendizaje de los objetos matemáticos como son: 1. Razonamiento, 2. Planteamiento y Resolución de problemas, 3. Comunicación, 4. Modelación y 5. Elaboración, comparación y ejercitación de procedimientos. Los tres primeros se acercan más a las competencias definidas por el Icfes. Ver Acevedo y col. (2007).

10. Con referencia a los procesos cognitivos específicos que desarrollan el pensamiento matemático y los sistemas propios de las matemáticas se plantea que estos conocimientos se pueden organizar en cinco tipos de pensamiento: 1. Numérico y sistemas numéricos, 2, Espacial y sistemas geométricos, 3. Métrico y sistemas de medida, 4. Aleatorio y sistemas de datos, 5. Variacional y sistemas algebraicos. Las pruebas Saber 11 agrupan estos cinco tipos de pensamiento en tres componentes: Numérico- variacional, Geométrico-métrico y Aleatorio. Ver Acevedo y col. (2007) para la conceptualización del área de matemáticas en la prueba Saber 11.
} 
Tabla 1.

\section{Competencias en Matemáticas}

\begin{tabular}{l|l}
\hline Competencias & \multicolumn{1}{c}{ Descripción } \\
\hline Comunicación & $\begin{array}{l}\text { Se refiere a la capacidad de identificar la coherencia de una idea respecto a } \\
\text { los conceptos matemáticos expuestos en una situación o contexto determinado; } \\
\text { usar diferentes tipos de representación; y describir relaciones matermáticas } \\
\text { a partir de una tabla, una gráfica, una expresión simbólica o una situación } \\
\text { descrita en lenguaje natural. Dentro de esta competencia también se evalúa } \\
\text { la habilidad para manipular proposiciones y expresiones que contengan } \\
\text { símbolos y fórmulas, es decir, el uso y la interpretación del lenguaje matemático. }\end{array}$ \\
\hline Razonamiento & $\begin{array}{l}\text { Se relaciona con la identificación y uso de estrategias y procedimientos para } \\
\text { tratar situaciones problema, la formulación de hipótesis y conjeturas y exploración } \\
\text { de ejemplos y contraejemplos, la identificación de patrones y la generalización } \\
\text { de propiedades. }\end{array}$ \\
\hline $\begin{array}{l}\text { Solución de } \\
\text { Problemas }\end{array}$ & $\begin{array}{l}\text { Se refiere a la capacidad de plantear y resolver problemas a partir de contextos } \\
\text { matemáticos y no matemáticos, de traducir la realidad a una estructura matemática } \\
\text { y de verificar e interpretar resultados a la luz de un problema, de manera que se } \\
\text { generalicen soluciones y estrategias que resuelvan nuevas situaciones. }\end{array}$ \\
\hline
\end{tabular}

Fuente: ICFES (2011)

De otra parte, en lo referente a los componentes evaluados, como ya mencionamos, la prueba de matemáticas pretende evaluar cinco tipos de pensamiento matemático que han sido agrupados en tres grandes componentes, como se muestra en la Tabla 2.

Tabla 2 .

\section{Competencias en Matemáticas}

Alude al significado del número y sus diferentes usos; a la estructura del sistema de numeración; al significado y utilización de las operaciones, así como a la comprensión de sus propiedades y las relaciones sí; al reconocimiento de regularidades y patrones; a la identificación de variables; a la descripción de fenómenos de cambio y dependencia; a la variación en contextos aritméticos y geométricos; y al concepto de función.

Numéricovariacional

Geométricométrico
Está relacionado con la construcción y manipulación de representaciones de objetos bidimensionales y tridimensionales, además de sus características, relaciones y transformaciones. También se refiere a la comprensión del espacio y el plano a través de la observación de patrones y regularidades, así como al razonamiento geométrico y a la solución de problemas de medición (longitud, área, volumen, capacidad, masa, tiempo, entre otras) a partir de la selección de unidades, patrones e instrumentos pertinentes.
Indaga por la lectura, representación e interpretación de datos extraídos de contextos no matemáticos (encuestas, resultados de experimentos, entre otros); el análisis de diversas formas de representación de información númerica; la elaboración de conjeturas sobre regularidades y tendencias presentadas en fenómenos estadísticos y probabilísticos; y el uso de medidas de centralización, posición, dispersión y forma. 
Es claro que estas descripciones de las competencias presuponen que los estudiantes son capaces de comprender los distintos registros semióticos de representación que les permiten entender la pregunta y llevar a cabo los distintos cambios de representación dentro del mismo registro de representación (tratamientos) como entre distintos registros de representación (conversiones). El manejo de las distintas formas de representación de los objetos matemáticos, así como de sus cambios de representación, permite evaluar si el estudiante entiende o no un concepto o conjunto de conceptos. Más aún, la prueba busca evaluar si el estudiante entiende los objetos matemáticos en sí mismos, es decir, si es capaz de generalizar y "traducir a la realidad" los distintos problemas y resolverlos en contextos matemáticos y no matemáticos.

Es así como la no respuesta a una pregunta puede ser evidencia de una dificultad para entender distintas formas de representación, es decir, dificultades para entender incluso el enunciado ${ }^{11}$. De igual manera, la respuesta incorrecta puede mostrar no solo dificultades para entender un registro de representación, sino dificultades en los cambios de representación que, en últimas, refleja problemas en los procesos de enseñanza y de aprendizaje.

Las Figuras 2 y 3 una vez más evidencias las dificultades en los procesos de enseñanza y aprendizaje de los objetos matemáticos. Como se mencionó anteriormente, el bajo porcentaje de respuesta al ítem para cada una de las competencias refleja las dificultades del proceso de enseñanza y aprendizaje, más aún muestra la necesidad de un mayor análisis de los enunciados que conforman el examen con el fin de entender cuáles son las principales dificultades de los estudiantes asociados con procesos de cambios de representación.

Figura 2. Distribución de Respuesta al Item. Competencia Comunicativa
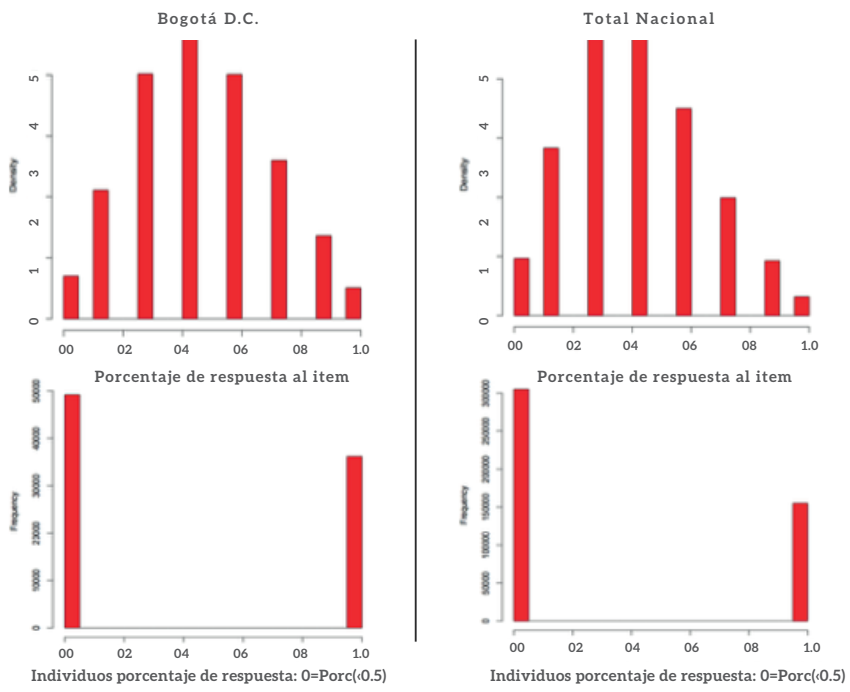

Fuente ICFES. Cálculos del Autor

11. Como no hemos tenido acceso a los enunciados mismos, es claro que partimos del supuesto de que los enunciados están bien diseñados y de que su grado de complejidad puede estar reflejado es en el tipo de representación escogido en el momento de elaborar la pregunta. De igual manera, partimos del supuesto de que el enunciado está asociado a la competencia que quiere medir, como ha sido definida por el ICFES. 


\subsection{Características Individuales}

Bajo el supuesto de que los individuos no tienen dificultades de aprendizaje o discapacidades que afecten directamente el aprendizaje de los objetos matemáticos, en esta sección queremos hacer énfasis en aquellas características propias del individuo que pueden afectar su desempeño. Dentro de aquellos factores individuales que afectan los procesos de enseñanza y aprendizaje y que se reflejan en la respuesta (correcta o incorrecta) o la no respuesta a un ítem, encontramos en primer lugar, la comprensión del enunciado al que se enfrenta el alumno.

En efecto, el desempeño en un área como el lenguaje es una condición que afectará el desempeño en matemáticas. Si un estudiante no entiende lo que se le está preguntando, más aún, cuando el enunciado al que se enfrenta es multi-registro, es decir, que combina diversos tipos de registros de representación (lenguaje natural, símbolos matemáticos, representaciones gráficas, etc.) es difícil pensar que un individuo se atreva a enfrentar una pregunta que ni siquiera entiende. Si los individuos tienen un bajo desempeño en lenguaje, es muy probable que su desempeño en matemáticas sea igualmente bajo. Esa es pues una hipótesis que vamos a contrastar.

Figura 3. Distribución de Respuesta al Item. Competencia Argumentativa

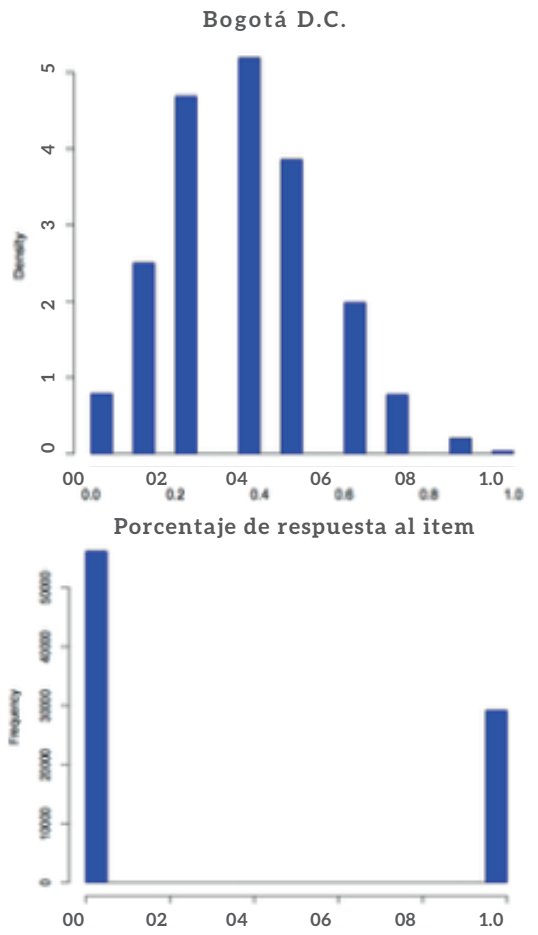

Individuos porcentaje de respuesta: $0=\operatorname{Porc}(<0.5)$
Total Nacional

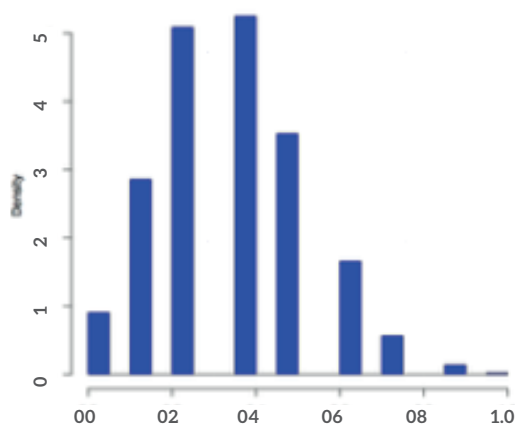

Porcentaje de respuesta al item

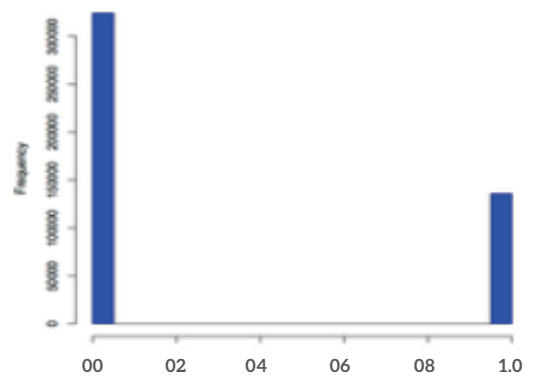

Individuos porcentaje de respuesta: $0=\operatorname{Porc}\left({ }^{0} 0.5\right)$

Fuente ICFES. Cálculos del Autor 


\subsection{El capital social}

El concepto de capital social ha sido recientemente utilizado como determinante del un mejor desempeño educativo en la educación superior y en la vida profesional, ver Ferrante y Sabatini (2007), Daily, Eugene y Prewitt (2007), Brown, Flick y Williamson (2005). Putnam (1994), Woolcock (2000) y Schuller (2000) definen el capital social como un concepto multidimensional que incluye características de redes sociales de vida, normas, confianza, que permite a los individuos que cuentan con dicho capital social actuar en diversos contextos de manera efectiva para perseguir objetivos comunes, ya sea individuales o de grupo. Putnam (1994) distingue dos tipos de componentes, el capital social que une a un individuo a un grupo, que forma un cierto tipo de pertenencia, así como el capital social que vincula y crea puentes entre diversos grupos sociales heterogéneos. Stanton-Salazar y Dornbush (1995)enfatizan cómo las diferencias en capital social profundizan las desigualdades.

Desde nuestra perspectiva, hacemos referencia al capital social como el conjunto de redes sociales y familiares, que implican unas normas y hábitos que estimulan el trabajo académico en general y el aprendizaje de las matemáticas en particular. Es así como el capital social está estrechamente vinculado con el capital humano (Schuller (2000)). En particular estamos asociando ese conjunto de redes y de hábitos que pueden ser aprendidos en un ambiente donde los padres y el entorno familiar y social valoran los procesos educativos y la formación de capital humano. Estamos interesados en aquellas variables que directa o indirectamente están asociadas a un alto nivel de capital humano que permite acceder, potenciar y formar el capital social de un individuo.

Cuando nos referimos al capital social, queremos hacer énfasis al ámbito familiar en el cual el individuo vive y que de manera directa e indirecta afecta los procesos de enseñanza y aprendizaje. Entre los factores que reflejan dicha acumulación de capital social está el nivel educativo de los padres o al menos del padre que permanece más tiempo con el estudiante.

Otro elemento que refleja este proceso de acumulación de capital social por parte de la familia es la presencia o no de un biblioteca o un conjunto de libros en el hogar, es decir, aquellos aspectos que contribuyen a la apropiación familiar del acervo cultural de la ciencia de su sociedad. Si la familia es educada y esto se refleja en la existencia de un conjunto de instrumentos que permiten el aprendizaje (libros, computador, materiales) es claro que dicho capital social se reflejará en el aprendizaje y por tanto en el desempeño en pruebas estandarizadas.

En nuestro caso, la otra variable proxy que podemos encontrar es el tener computador o no en el hogar. Si bien esta variable pude reflejar aspectos socieconómicos como el tener horno en la casa o las características de la vivienda, es claro que el computador y en particular las herramientas computacionales asociadas a él contribuyen directamente al aprendizaje y al desempeño académicos. Diversos estudios sobre impacto de programas como Computadores para Educar y el impacto del uso de tecnologías en el currículo de matemáticas así lo demuestran.

\subsection{El entorno escolar}

Además de la infraestructura física y la planta docente que muestran cómo el desempeño escolar es claro que el rendimiento escolar depende de los hábitos escolares, del conjunto de normas que permiten el funcionamiento día a día de una escuela y que forman, 
como dice Brousseau (1986) parte del "medio" en el cual se desarrolla los procesos de enseñanza y aprendizaje. El efecto plantel ha sido ampliamente documentado y, junto con los aspectos socieconómicos, muestran su impacto sobre el desempeño académico, así como en otros indicadores como la deserción, la repitencia. Los distintos trabajos para Colombia sobre desempeño en educativo muestran el efecto plantel como un factor determinante (Barrera (2005), Barrientos y Gaviria (2001a), Barrientos y Gaviria (2001b), Barrientos y Gaviria (2001c), Becerra, González y Sarmiento (2000)). McGowen (2009)hace una revisión detallada de los distintos estudios sobre entorno escolar y desempeño académico en las escuelas.

El entorno escolar juega un papel central en la medida en que es en ese ámbito en particular donde se desarrollan principalmente lo que Brousseau (1986) denomina las situaciones didácticas y dentro de estas, las situaciones a-didácticas. Es claro que las características del colegio, así como la existencia o no de bibliotecas, laboratorios, y una planta de profesores cualificados influyen sustancialmente en el desempeño académico en general, y esto debe reflejarse en el desempeño de los estudiantes en las distintas pruebas.

Sin embargo, es claro que, si bien estos factores han sido ampliamente documentados, sus efectos directos en términos de mejoras en las prácticas educativas son muy difusos, así como los esfuerzos de plantear políticas que conduzcan a mejorar el desempeño son muy limitadas en su implementación. Es decir, que los colegios públicos y los privados muestran desempeños sustancialmente distintos, o que los estudiantes de menores ingresos muestran desempeños académicos significativamente menores que aquellos con ingresos altos, no permite plantear por si mismo soluciones específicas en términos de políticas educativas.

\section{ESPECIFICACIÓN DEL MODELO HGLM EN DOS NIVELES}

La estrategia para la estimación del modelo HGLM multinivel sigue la metodología de Hendry (1995) de partir de un modelo general y analizar el conjunto de modelos que se pueden derivar al imponer restricciones sobre los parámetros. En los HGLM en dos o tres niveles, el resultado esperado en cada uno de los niveles puede ser representado por una función de regresión lineal sobre los coeficientes y se supone que los efectos aleatorios de cada nivel tienen una distribución normal. Sin embargo, el supuesto de normalidad no es adecuado y no es posible hacer transformaciones para lograr una distribución aproximadamente normal, como es el caso cuando el resultado es binario. El uso de una estimación estándar en el primer nivel es inapropiada debido a tres razones:

- Los valores del modelo estimado en el nivel 1 del modelo estándar no toman valores restringidos, como si es el caso de un modelo binario, donde el modelo estimado es interpretado como la probabilidad de $\mathrm{Y}=1 \mathrm{E}(0,1)$, es decir, $\operatorname{Prob}(\mathrm{Y}=1)=$ $\mathrm{F}\left(\mathrm{B}^{\wedge} \mathrm{X}\right)$, con $\mathrm{F}(\bullet)$ la distribución de probabilidad.

- Como el modelo estimado en el nivel 1 únicamente puede tomar dos valores, no puede tener una distribución normal.

- La varianza de los efectos aleatorios en el nivel 1 no puede ser homogénea. 
En nuestro caso, cada uno de los modelos que se deben especificar a nivel de pregunta tiene esta característica, e indica si el porcentaje de respuesta al ítem para el j-ésimo componente de matemáticas por parte del individuo i-ésimo fue mayor o igual al nivel de suficiencia predeterminado. (Yij = 1 si el porcentaje de respuesta al ítem es mayor o igual a $50 \%$; Yij = 0 en caso contrario).

Se especificó un HGLM en dos niveles. La especificación del primer nivel consiste de tres partes:

\section{Modelo muestral nivel-1}

Tenemos que $\mathrm{Y}_{\mathrm{ij}}$ corresponde a la respuesta correcta al presentar la prueba Saber 11 . Sea $\varphi$ la probabilidad de que la respuesta sea correcta. Formalmente, tenemos que el modelo Binomial particular para nuestro caso es una distribución Bernoulli, tal que:

$$
Y_{i j} \mid \varphi_{i j} \sim B\left(1, \varphi_{i j}\right)
$$

Los parámetros del modelo Binomial para una distribución Bernoulli implica una oportunidad y la probabilidad de éxito en dicho intento. La media y la varianza respectiva- mente está dada por:

$$
E\left(Y_{i j} \mid \varphi\right)=\varphi_{i j} ; \operatorname{Var}\left(Y_{i j} \mid \varphi_{i j}\right)=\varphi_{i j}\left(1-\varphi_{i j}\right)
$$

\section{Función de enlace nivel-1}

Debemos transformar el valor estimado $\varphi_{\text {ij }}$ de manera que mantenga el rango de los valores estimados, para el caso Binomial, la función de enlace está dada por:

$$
\eta_{i j}=\log \left(\frac{\varphi_{i j}}{1-\varphi_{i j}}\right)
$$

\section{Modelo estructural del nivel-1}

Finalmente, el valor estimado transformado $n_{\mathrm{ij}}$, en el rango de valores restringidos por el modelo binomial, es relacionado con los predictores o variables que explican, en nuestro caso particular, la respuesta correcta a la j-ésima pregunta del componente de matemáticas de la prueba Saber 11 por parte del i-ésimo sujeto, tenemos así que el modelo lineal estructural para $n$ factores explicativos está dado por:

$$
\eta_{i j}=\beta_{0 j}+\sum_{h=1}^{n} \beta_{h j} X_{h i j}+\sum_{k=1}^{m} \sum_{h=1}^{n} \beta_{k i j} X_{k i j}
$$

Para esta especificación particular del modelo hemos dividido nuestro conjunto de variables explicatorias en dos grupos. El primer grupo, el vector (X) hace referencia a las características del estudiante: Genero, Edad, Desempeño en el área de lenguaje, estudiante trabaja. Es importante resaltar cómo el manejo de la lengua natural es esencial en la comprensión y argumentación de enunciados matemáticos. 
El segundo grupo, el vector (Z) hace referencia a tres aspectos del ambiente que pueden afectar su proceso de aprendizaje y por tanto su desempeño, las variables incluidas son: Nivel educativo de los Padres, si se tiene computador, jornada escolar a la que asiste (completa, mañana, tarde, noche, sábados).

Observe que el modelo podría incluir un conjunto de variables exógenas. En nuestro caso, algunas variables que capturen las características de las preguntas.

En general, los distintos estudios centran su atención en el ambiente del colegio, es decir, en las características del colegio. Una primera parte de nuestro estudio ha buscado explorar el ambiente inmediato, distinto del las interacciones directas o indirectas del ámbito netamente escolar (desempeño promedio de los compañeros, si el colegio es público o privado) que afectan el proceso de aprendizaje, de las cuales tenemos información.

\section{Nivel-2}

El análisis para el segundo nivel sigue la especificación estándar.

$$
\beta_{q j}=\gamma_{q 0}+\sum_{s=1}^{s_{q}} \gamma_{q s} W_{s j}+u_{q j}
$$

El segundo nivel se especificó combinando dos criterios: la naturaleza de los colegios (público o privado) y la clasificación de calidad realizada por el Icfes de acuerdo con los resultados en la Prueba Saber11, es así como se creó la variable plantelcalidad como aparece en la Tabla $3 .^{12}$

Tabla 3.

Grupos para el 2o Nivel del HGLM

\begin{tabular}{cll}
\hline Plantelcalidad & Naturaleza & \multicolumn{1}{|c}{ Calidad } \\
\hline 1 & No oficial & Muy superior \\
2 & No oficial & Superior \\
3 & No oficial & Alto \\
4 & No oficial & Medio \\
5 & No oficial & Bajo \\
6 & No oficial & Inferior \\
7 & No oficial & Muy inferior
\end{tabular}

12. Nótese que esta definición introduce una autocorrelación entre los promedios del año anterior y los del siguiente, pero crea una proxy aceptable de calidad del plantel, así no sea ordinal. Esto se debe a que la interacción entre calidad del colegio y naturaleza no captura la ordinalidad, es decir, que un colegio Oficial muy superior posiblemente pueda seguir en escala a un colegio No Oficial de nivel Superior o Alto. Este análisis requiere un estudio aparte. 
Tabla 3.

Grupos para el 2o Nivel del HGLM

\begin{tabular}{cll}
\hline Plantelcalidad & Naturaleza & \multicolumn{1}{c}{ Calidad } \\
\hline 1 & Oficial & Muy superior \\
2 & Oficial & Superior \\
3 & Oficial & Alto \\
4 & Oficial & Medio \\
5 & Oficial & Bajo \\
6 & Oficial & Inferior \\
7 & Oficial & Muy inferior \\
\hline
\end{tabular}

Fuente: Elaboración propia.

En el segundo nivel, se buscaría principalmente modelar los coeficientes asociados a factores relacionados directamente con el ambiente familiar como el nivel educativo de los padres y el que haya o no computador en el hogar.

\section{ESTIMACIÓN DEL MODELO}

Para la estimación del modelo se utilizaron las bases de datos proporcionadas por el ICFES para los estudiantes que el segundo semestre de 2009 presentaron las pruebas Saber 11. Se utilizaron cuatro bases de datos:

- Base a nivel de pregunta (35 Preguntas en 2009) por estudiante, en el área de Matemáticas

- Base con características socieconómicas por estudiante

- Base de características de los colegios (Algunas variables son promedios de los datos a nivel de estudiante)

- Base del índice socieconómico del colegio para 2009, construido por el Icfes a partir de la información socieoeconómica de los estudiantes

Las bases fueron organizadas a nivel de estudiante y luego, para poder capturar el efecto de respuesta al item por competencias, se construyeron tres variables para capturar el porcentaje de respuesta al ítem por competencia (Comunicación, Razonamiento y Solución de Problemas). Luego se procedió a fijar un criterio para medir al suficiencia por estudiante a cada uno de los componentes, el criterio definitivo fue 0.5 , es decir, si un estudiante responde el 50 \% o más de las preguntas relacionadas con el componente de solución de problemas, diremos que alcanza un nivel de suficiencia en dicha competencia. Como se mostró anteriormente en las Figuras 1, 2 y 3 la media de los estudiantes está al rededor del 40\%. Un criterio de suficiencia bajo, sesga los 
resultados hacia un despeño pobre, mientras que un criterio de suficiencia muy alto generó dificultades en la estimación. Si bien se corrió el ejercicio para varios criterios, sólo se reportan aquellos para los cuales el criterio de suficiencia fue de 0.5.

La estimación se llevó a cabo en dos niveles. El primer nivel corresponde a la información de desempeño del individuo en las pruebas de competencias, mientras que para el segundo nivel, se tuvo en cuenta los criterios de calidad y naturaleza de los colegios para conformar los 14 grupos correspondientes a la variable plantelcalidad.

Inicialmente se estimaron dos tipos de modelos, el modelo no condicional y los modelos que capturan el efecto de la media de acuerdo a los cuatro niveles de análisis definidos en la segunda parte.

Finalmente, se estimo el efecto total (cambio en los interceptos y en las pendientes) de las variables explicatorias seleccionadas a partir de las correlaciones encontradas.

Todos los modelos se corrieron en el programa R-Statistic ${ }^{13}$. El programa base para la estimación del modelo, así como la salida de los resultados está entre los archivos que forman parte del documento.

\section{Efectos aleatorios: Modelos con efectos totales (pendiente y medias)}

Para la estimación del desempeño por competencia condicionada a los distintos conjuntos de factores se estimó el siguiente modelo que presentamos a continuación siguiendo la notación de Bryk y Raudenbush (2002) y Bliesse (2003)

Uno de los elementos centrales de los HGLM es la de poder separar los efectos fijos que afectan el intercepto y los efectos aleatorios asociados a la pendiente. Formalmente, nuestro modelo estructural con efectos totales corresponde a:

$$
\begin{gathered}
\text { Competencia }(k)_{i j}=\beta_{0 j}+\beta_{m j} X_{m j}+r_{i j} \\
\beta_{0 j}=\gamma_{00}+\sum_{n=1}^{q} \gamma_{0 n} W_{n j}+u_{0 j} \\
\beta_{m j}=\gamma_{m j}+\gamma_{l j} Z_{l j}+u_{m j} \forall m=1, \ldots, p
\end{gathered}
$$

Observe que $\mathrm{X}$ corresponde al conjunto de variables dependientes asociadas con las características del individuo o de su entorno familiar, mientras que las W corresponde al conjunto de variables asociadas al segundo nivel, a nivel de escuela. Las variables utilizadas en la estimación de los modelo aparecen en el Tabla 4. 
Tabla 4.

Variables Dependientes Modelo HGLM

\begin{tabular}{l|l}
\hline Características & \multicolumn{1}{c}{ Variables } \\
\hline \multirow{2}{*}{ Individuo } & Género (Femenino = 1) \\
& Edad \\
& Desempeño Lenguaje \\
& Estudiante Trabaja (Si=1) \\
& Nivel educativo de la Madre \\
& Tipo empleo del Padre \\
& Computador (Si=1) \\
& Ingreso Familiar \\
& Jornada (Completa, Mañana, Tarde, Noche, Sábado) \\
& \\
& Desempeño Promedio del Plantel en Matemáticas \\
& Índice Socioeconómico del Plantel (2009) \\
& Tipo Plantel por Género (Femenino, Masculino, Mixto) \\
& Naturaleza del Plantel (No Oficial, Oficial) \\
& Calidad del Plantel (Muy Superior, Superior, Alto, Medio, \\
& \\
\hline &
\end{tabular}

Fuente: ICFES, Elaboración propia.

En forma reducida, el modelo estructural puede ser expresado como:

$$
\begin{aligned}
& \operatorname{Competencia}(k)_{i j}=\gamma_{00}+\gamma_{m j} X_{m j}+\sum_{n=1}^{q} \gamma_{0 n} W_{n j}+ \\
& \sum_{m=1}^{p} \gamma_{m j} X_{m j} Z_{m j}+u_{o j}+\sum_{m=1}^{p} \gamma_{m j} X_{m j} u_{m j}+u_{m j}+r_{i j}
\end{aligned}
$$

Donde el primer reglón de la ecuación captura el efecto fijo, mientras que, los términos del segundo reglón capturan los efectos aleatorios.

Los efectos aleatorios son capturados por tres conjuntos de variables: interacciones $(\mathrm{X} \bullet \mathrm{Z})$; efectos aleatorios que afectan características individuales ( $\mathrm{X} \bullet \mathrm{u}$ ) (efectos aleatorios sobre la pendiente); $y$ los efectos aleatorios de pendiente $\sin$ interacciones $\left(\mathrm{u}_{\mathrm{mj}}\right)$. 


\section{RESULTADOS POR COMPETENCIAS PARA BOGOTÁ Y NIVEL NACIONAL}

En esta sección se hace una presentación detallada de los modelos que capturan los efectos fijos y los efectos aleatorios para Bogotá y el nivel nacional ${ }^{14}$.

\section{Características del individuo}

Frente a las características del individuo, para las tres competencias, los resultados son significativos y en la misma dirección tanto a nivel Nacional como a nivel de Bogotá. En efecto, el que a los estudiantes les vaya bien en el componente de lenguaje tiene una relación positiva y significativa con el desempeño en matemáticas para cada una de las componentes. Desde la perspectiva de nuestro marco teórico este resultado es muy relevante en la medida en que de manera directa e indirecta pone de relieve los tipos de registros de representación semiótica, así como los cambios de representación entre los mismos. El impacto es mayor para la competencia comunicativa.

De igual forma, existe una relación negativa entre el desempeño y la edad. Esto es comprensible y es muy probable que esté relacionado con un alto grado de repitencia en los cursos, lo que conlleva a que los estudiantes ya no se mantenga dentro de su cohorte para cada nivel educativo. Estos estudiantes deben trabajar y terminan a mayor edad la educación media, lo que afecta su desempeño y la culminación de sus estudios de secundaria. Si bien a medida que el individuo crece, una mayor edad puede reflejar una mayor capacidad cognitiva para aprender y entender conceptos, de igual manera, entre mayor sea la edad, existirá una mayor dificultad para entender dichos conceptos si la extra-edad refleja repitencia, entrada tarde al sistema escolar, o períodos de abandono de éste, lo cual apunta más bien a que no se ha tenido una formación buena y progresiva que le hubiera permitido al bachiller en cuestión haber progresado en el entendimiento y la relación entre distintos objetos matemáticos.

Finalmente, el hecho que un estudiante trabaje a la vez que estudia o si está trabajando cuando presenta la prueba Saber 11 puede estar asociado a que hace algún tiempo terminó el bachillerato sin graduarse o está repitiendo el examen por haber obtenido antes un puntaje muy bajo. De igual manera puede reflejar el "fracaso" escolar repetitivo que lo ha expulsado de su cohorte, así como la necesidad de dedicar parte del tiempo, que otros estudiantes de su edad dedican a estudiar, a actividades laborales. Los resultados muestran una correlación negativa en particular con las competencias argumentativa y de solución de problemas. Allí es menor el número de estudiantes cuyo porcentaje de respuesta al ítem es mayor del $50 \%$.

De igual manera, en el caso de las mujeres, para todas las componentes, su desempeño es menor que el de los hombres. Esto debe plantear un estudio más detallado que permita analizar las causas y desarrollar estrategias pedagógicas para la enseñanza y el aprendizaje de las matemáticas en las mujeres.

14. En el estudio se estimaron 21 modelos multinivel para Bogotá y 21 modelos para el nivel nacional. Estos modelos se corrieron para varios niveles de suficiencia. Sólo se reportan el Modelo completo de Efectos de Media Condicionada y el Modelo que captura los Efectos Totales (Efectos fijos y aleatorios) 


\section{Características de capital social}

Al analizar los resultados de las variables de capital social, los resultados NO son significativos para todo el conjunto de variables utilizadas. En particular el nivel educativo de la madre o el nivel de ocupación del padre, que son las variables directas para capturar éste efecto no son del todo significativas y su coeficiente es cercano a cero.

Mientras que las variables indirectas, como el número de miembros de la familia, si hay computador en la casa y el ingreso familiar, son significativas para todas las componentes y su signo es el esperado. Para todas las componentes se encontró que la existencia o no de computador en la casa tiene un impacto positivo con relación al desempeño en cada una de las pruebas. Más aún, dicho impacto es mayor a Nivel Nacional que para Bogotá. Esta variable es la proxy que busca relacionar de manera imperfecta el impacto de un ambiente cultural al rededor del individuo como el acceso a una biblioteca. Podríamos conjeturar que la existencia del computador permite de alguna manera desarrollar la capacidad del individuo de pasar de un registro semiótico gráfico a uno escrito en lengua natural y viceversa, lo que facilitaría el desarrollo de habilidades para entender los objetos matemáticos, sus distintos tipos de representación y los cambios de representación. Para la búsqueda de información, el computador con internet es aún más potente que el acceso a una biblioteca. Por esta razón se recomienda introducir el factor con o sin acceso a internet.

De igual manera, el número de miembros en el hogar, muestra una relación negativa con el desempeño en cada una de las componentes. En las sociedades en las cuales el proceso de transición demográfica ha terminado o está en proceso la tasa de fecundidad y el número de hijos está relacionado inversamente con el nivel educativo de los padres y el conjunto de oportunidades que se puede brindar cuando el hogar tiene un menor número de miembros.

En cuanto al ingreso familiar, que indirectamente está asociado, en la mayoría de los casos, a un mayor nivel educativo que se reflejan en un mejor ingreso, muestra una relación positiva con el desempeño en matemáticas. Este es un resultado estándar en los distintos estudios mencionados en la introducción.

Finalmente debemos mencionar, que la jornada (completa, mañana, tarde, noche y sábados) busca capturar de alguna manera los hábitos y rutinas en el proceso de aprendizaje de los estudiantes. Con respecto a esto es importante mencionar que para todas las competencias en Bogotá, las jornadas completa, mañana son significativas y tienen un impacto positivo sobre el desempeño y su rendimiento en promedio es mejor que con respecto a Jornada de la Tarde. Para el caso nacional, si bien todas las jornadas muestran una relación positiva y significativa, su efecto es mayor en las jornadas completa y mañana, que en las jornadas nocturnas y de los sábados. Esto está asociado, como mencionamos a los distintos hábitos y posibilidades de construir un capital social que brinda el entorno familiar. Los jóvenes que estudian en esas jornadas tienen un desempeño que es probable que esté asociado a factores de repitencia y deserción escolar, y de igual manera deben estar asociados a una mayor edad con relación a los estudiantes de jornadas completa o de la mañana. 
Tabla 5.

HGLM. Efectos Totales (Pendientes y Medias)

\begin{tabular}{|c|c|c|c|c|c|c|}
\hline & \multicolumn{3}{|c|}{ Bogotá D.C. } & \multicolumn{3}{|c|}{ Total Nacional } \\
\hline Variables & Comunicativa & Argumentativa & $\begin{array}{l}\text { Soluc. } \\
\text { Problemas }\end{array}$ & Comunicativa & Argumentativa & $\begin{array}{l}\text { Soluc. } \\
\text { Problemas }\end{array}$ \\
\hline Constante & $\begin{array}{l}-5,171^{* * *} \\
0,606\end{array}$ & $\begin{array}{l}-3,053^{* * *} \\
0,471\end{array}$ & $\begin{array}{l}-7,499^{* * *} \\
0,6,33\end{array}$ & $\begin{array}{l}-5,392^{* * *} \\
0,333\end{array}$ & $\begin{array}{l}-2,979^{* * *} \\
0,213\end{array}$ & $\begin{array}{l}-6,790^{* * *} \\
0,343\end{array}$ \\
\hline Género (Femenino=1) & $\begin{array}{l}-0,548^{* * *} \\
0,016\end{array}$ & $\begin{array}{l}-0,300^{* * *} \\
0,016\end{array}$ & $\begin{array}{l}-0,637^{* * *} \\
0,023\end{array}$ & $\begin{array}{l}-0,492^{* * *} \\
0,008\end{array}$ & $\begin{array}{l}-0,282^{* * *} \\
0,007\end{array}$ & $\begin{array}{l}-0,605^{* * *} \\
0,012\end{array}$ \\
\hline Edad & $\begin{array}{l}-0,042^{* * *} \\
0,003\end{array}$ & $\begin{array}{l}-0,028^{* * *} \\
0,003\end{array}$ & $\begin{array}{l}-0,045^{* * *} \\
0,006\end{array}$ & $\begin{array}{l}-0,035^{* * *} \\
0,001\end{array}$ & $\begin{array}{l}-0,020^{* * *} \\
0,001\end{array}$ & $\begin{array}{l}-0,033^{* * *} \\
0,003\end{array}$ \\
\hline $\begin{array}{l}\text { Puntaje Saber11 } \\
\text { en Lenguaje }\end{array}$ & $\begin{array}{l}0,053^{* * *} \\
0,009\end{array}$ & $\begin{array}{l}0,017^{* * *} \\
0,007\end{array}$ & $\begin{array}{l}0,068^{* * *} \\
0,010\end{array}$ & $\begin{array}{l}0,061^{* * *} \\
0,004\end{array}$ & $\begin{array}{l}0,004 \\
0,003\end{array}$ & $\begin{array}{l}0,043 \\
0,005\end{array}$ \\
\hline Estudiante Trabaja ( $\mathrm{Si}=1$ ) & $\begin{array}{l}0,009^{* * *} \\
0,004\end{array}$ & $\begin{array}{l}-0,019^{* * *} \\
0,004\end{array}$ & $\begin{array}{l}-0,005^{* * *} \\
0,006\end{array}$ & $\begin{array}{l}0,006^{* * *} \\
0,002\end{array}$ & $\begin{array}{l}-0,005^{* * *} \\
0,002\end{array}$ & $\begin{array}{l}-0,007^{* * *} \\
0,003\end{array}$ \\
\hline Nivel Educativo Madre & $\begin{array}{l}0,001 \\
0,003\end{array}$ & $\begin{array}{l}0,004 \\
0,003\end{array}$ & $\begin{array}{l}0,013^{* * * *} \\
0,004\end{array}$ & $\begin{array}{l}-0,001 \\
0,002\end{array}$ & $\begin{array}{l}0,001 \\
0,001\end{array}$ & $\begin{array}{l}0,005 \\
0,002\end{array}$ \\
\hline Nivel Educativo Padre & $\begin{array}{l}-0,001 \\
0,003\end{array}$ & $\begin{array}{l}0,001 \\
0,003\end{array}$ & $\begin{array}{l}0,018^{* * * *} \\
0,004\end{array}$ & $\begin{array}{l}-0,004 \\
0,001\end{array}$ & $\begin{array}{l}-0,001 \\
0,001\end{array}$ & $\begin{array}{l}0,002 \\
0,002\end{array}$ \\
\hline $\begin{array}{l}\text { Número de Miembros } \\
\text { en la Familia }\end{array}$ & $\begin{array}{l}-0,010^{* *} \\
0,004\end{array}$ & $\begin{array}{l}-0,009^{* *} \\
0,004\end{array}$ & $\begin{array}{l}-0,012^{* *} \\
0,006\end{array}$ & $\begin{array}{l}-0,021^{* *} \\
0,002\end{array}$ & $\begin{array}{l}-0,006^{* *} \\
0,002\end{array}$ & $\begin{array}{l}-0,018^{* *} \\
0,003\end{array}$ \\
\hline $\begin{array}{l}\text { Tiene Computador } \\
(\mathrm{Si}=1)\end{array}$ & $\begin{array}{l}0,020 \\
0,053\end{array}$ & $\begin{array}{l}0,023 \\
0,046\end{array}$ & $\begin{array}{l}0,163^{* *} \\
0,077\end{array}$ & $\begin{array}{l}0,051^{* *} \\
0,025\end{array}$ & $\begin{array}{l}-0,052^{* *} \\
0,021\end{array}$ & $\begin{array}{l}-0,042^{* *} \\
0,037\end{array}$ \\
\hline Ingreso Familiar & $\begin{array}{l}0,090^{* * *} \\
0,008\end{array}$ & $\begin{array}{l}0,029^{* * *} \\
0,008\end{array}$ & $\begin{array}{l}0,057^{* * *} \\
0,010\end{array}$ & $\begin{array}{l}0,099^{* * *} \\
0,004\end{array}$ & $\begin{array}{l}0,034^{* * *} \\
0,004\end{array}$ & $\begin{array}{l}0,080^{* * *} \\
0,006\end{array}$ \\
\hline $\begin{array}{l}\text { d_jornada_1 } \\
\text { (C=Completa) }\end{array}$ & $\begin{array}{l}0,245^{* * *} \\
0,042\end{array}$ & $\begin{array}{l}0,160^{* * *} \\
0,041\end{array}$ & $\begin{array}{l}0,303^{* * *} \\
0,067\end{array}$ & $\begin{array}{l}0,200^{* * *} \\
0,018\end{array}$ & $\begin{array}{l}0,134^{* * *} \\
0,017\end{array}$ & $\begin{array}{l}0,184^{* * *} \\
0,032\end{array}$ \\
\hline $\begin{array}{l}\text { d_jornada_2 } \\
\text { (M=Mañana) }\end{array}$ & $\begin{array}{l}0,227^{* * *} \\
0,039\end{array}$ & $\begin{array}{l}0,121^{* * *} \\
0,038\end{array}$ & $\begin{array}{l}0,262^{* * *} \\
0,064\end{array}$ & $\begin{array}{l}0,179^{* * *} \\
0,018\end{array}$ & $\begin{array}{l}0,112^{* * *} \\
0,016\end{array}$ & $\begin{array}{l}0,183^{* * *} \\
0,031\end{array}$ \\
\hline $\begin{array}{l}d_{\_} \text {jornada_4 } \\
(\mathrm{N}=\text { Noche })\end{array}$ & $\begin{array}{l}0,019 \\
0,101\end{array}$ & $\begin{array}{l}0,059 \\
0,099\end{array}$ & $\begin{array}{l}0,077 \\
0,172\end{array}$ & $\begin{array}{l}0,148^{* * *} \\
0,029\end{array}$ & $\begin{array}{l}0,060^{* *} \\
0,026\end{array}$ & $\begin{array}{l}0,140^{* *} \\
0,063\end{array}$ \\
\hline $\begin{array}{l}\text { d_jornada_5 } \\
\text { ( } \mathrm{S}=\text { Sábado) }\end{array}$ & $\begin{array}{l}0,123 \\
0,040\end{array}$ & $\begin{array}{l}0,050 \\
0,040\end{array}$ & $\begin{array}{l}0,117^{*} \\
0,067\end{array}$ & $\begin{array}{l}0,120^{* * *} \\
0,019\end{array}$ & $\begin{array}{l}0,081^{* * *} \\
0,018\end{array}$ & $\begin{array}{l}0,122^{* * *} \\
0,033\end{array}$ \\
\hline $\begin{array}{l}\text { Puntaje promedio } \\
\text { Colegio Matemáticas }\end{array}$ & $\begin{array}{l}0,162^{*} \\
0,093\end{array}$ & $\begin{array}{l}0,091 \\
0,068\end{array}$ & $\begin{array}{l}0,332^{* * *} \\
0,093\end{array}$ & $\begin{array}{l}0,239^{* * *} \\
0,044\end{array}$ & $\begin{array}{l}0,054^{* * *} \\
0,032\end{array}$ & $\begin{array}{l}0,338^{*} \\
0,049\end{array}$ \\
\hline $\begin{array}{l}\text { índice Socioeconómico } \\
\text { Colegio }\end{array}$ & $\begin{array}{l}0,016 \\
0,029\end{array}$ & $\begin{array}{l}-0,001 \\
0,028\end{array}$ & $\begin{array}{l}0,027 \\
0,043\end{array}$ & $\begin{array}{l}0,041^{* * *} \\
0,010\end{array}$ & $\begin{array}{l}-0,032^{* * *} \\
0,009\end{array}$ & $\begin{array}{l}0,006^{* * *} \\
0,015\end{array}$ \\
\hline $\begin{array}{l}\text { d_colgenero1 } \\
(\text { Femenino = 1) }\end{array}$ & $\begin{array}{l}0,273^{* * *} \\
0,068\end{array}$ & $\begin{array}{l}0,022 \\
0,053\end{array}$ & $\begin{array}{l}0,192^{* * *} \\
0,059\end{array}$ & $\begin{array}{l}0,257^{* * *} \\
0,036\end{array}$ & $\begin{array}{l}0,030 \\
0,045\end{array}$ & $\begin{array}{l}0,209 \\
0,036\end{array}$ \\
\hline $\begin{array}{l}\text { d_colgenero2 } \\
(\text { Masculino = 1) }\end{array}$ & $\begin{array}{l}-0,153^{* * *} \\
0,056\end{array}$ & $\begin{array}{l}-0,043^{* * *} \\
0,083\end{array}$ & $\begin{array}{l}-0,038 \\
0,064\end{array}$ & $\begin{array}{l}-0,101 \\
0,057\end{array}$ & $\begin{array}{l}-0,138^{* *} \\
0,062\end{array}$ & $\begin{array}{l}-0,113^{* *} \\
0,044\end{array}$ \\
\hline
\end{tabular}


Tabla 5.

HGLM. Efectos Totales (Pendientes y Medias)

\begin{tabular}{|c|c|c|c|c|c|c|}
\hline \multirow[b]{2}{*}{ Variables } & \multicolumn{3}{|c|}{ Bogotá D.C. } & \multicolumn{3}{|c|}{ Total Nacional } \\
\hline & Comunicativa & Argumentativa & $\begin{array}{l}\text { Soluc. } \\
\text { Problemas }\end{array}$ & Comunicativa & Argumentativa & $\begin{array}{l}\text { Soluc. } \\
\text { Problemas }\end{array}$ \\
\hline d_colnaturaleza2 & 0,057 & 0,019 & $0,133^{* * * *}$ & $0,194^{* * *}$ & $0,088^{* * *}$ & $0,041^{* * *}$ \\
\hline (Ofiical = 1) & 0,045 & 0,032 & 0,040 & 0,046 & 0,018 & 0,021 \\
\hline d_colcalidad1 & 14,999 & 21,141 & $-14,547$ & $-0,420$ & $0,875^{* *}$ & $0,960^{* *}$ \\
\hline (Muy superior $=1$ ) & 6512,087 & 6518,079 & 10839,575 & 0,545 & 0,288 & 0,482 \\
\hline d_colcalidad2 & 0,406 & 0,422 & 0,351 & $-0,349^{* *}$ & $0,552^{* * *}$ & $0,185^{* * *}$ \\
\hline (Superior = 1) & 0,347 & 0,293 & 0,428 & 0,138 & 0,089 & 0,070 \\
\hline d_colcalidad3 & $0,316^{*}$ & $0,160^{*}$ & $0,153^{*}$ & $-0,421^{* *}$ & $0,532^{* * *}$ & $0,092^{* * *}$ \\
\hline (Alto = 1) & 0,149 & 0,106 & 0,122 & 0,106 & 0,086 & 0,057 \\
\hline d_colcalidad4 & $0,340^{*}$ & $0,252^{* *}$ & $0,175^{* *}$ & $0,165^{* *}$ & $0,481^{* * *}$ & $0,081^{* * *}$ \\
\hline$($ Medio = 1) & 0,141 & 0,085 & 0,094 & 0,100 & 0,085 & 0,050 \\
\hline d_colcalidad4 & $0,415^{* *}$ & $0,239^{* *}$ & $0,217^{*}$ & 0,024 & $0,460^{* * *}$ & $0,086^{* * *}$ \\
\hline$($ Bajo = 1) & 0,139 & 0,080 & 0,087 & 0,097 & 0,086 & 0,044 \\
\hline d_colcalidad5 & $0,481^{* *}$ & $0,291^{* *}$ & $0,264^{* *}$ & 0,021 & $0,385^{* * *}$ & $0,045^{* * *}$ \\
\hline (Inferior = 1) & 0,144 & 0,052 & 0,090 & 0,098 & 0,092 & 0,040 \\
\hline Lenguaje & $0,002^{* *}$ & 0,001 & 0,000 & $0,003^{* * *}$ & $0,003^{* * *}$ & $0,004^{* * *}$ \\
\hline Computador & 0,001 & 0,001 & 0,001 & 0,000 & 0,000 & 0,001 \\
\hline Nivel Educativo & 0,000 & 0,000 & $-0,000^{* * *}$ & 0,000 & 0,000 & 0,000 \\
\hline $\begin{array}{l}\text { Madre: Ocupación } \\
\text { Padre }\end{array}$ & 0,000 & 0,000 & 0,000 & 0,000 & 0,000 & 0,000 \\
\hline $\begin{array}{l}\text { Computador: Índice } \\
\text { Socioeconómico }\end{array}$ & $\begin{array}{l}-0,041^{* * *} \\
0,011\end{array}$ & $\begin{array}{l}-0,011 \\
0,010\end{array}$ & $\begin{array}{l}-0,049^{* * *} \\
0,015\end{array}$ & $\begin{array}{l}-0,046^{* * *} \\
0,005\end{array}$ & $\begin{array}{l}-0,015^{* * *} \\
0,004\end{array}$ & $\begin{array}{l}-0,040^{* * *} \\
0,006\end{array}$ \\
\hline Lenguaje: Matemáticas & $0,004^{* * *}$ & $0,003^{* * *}$ & 0,000 & $0,003^{* * *}$ & $0,005^{* * *}$ & $0,002^{* * *}$ \\
\hline Colegio & 0,001 & 0,001 & 0,001 & 0,001 & 0,000 & 0,001 \\
\hline No. Observaciones & 85099 & 85099 & 85099 & 414550 & 414550 & 414550 \\
\hline No. Grupos & 13 & 13 & 13 & 14 & 14 & 14 \\
\hline
\end{tabular}

Robust standard errors in brackets

*** $\mathrm{p}<0,01, \quad{ }^{* *} \mathrm{p}<0,05, \quad{ }^{*} \mathrm{p}<0,01$

Fuente: ICFES, Cálculos del autor. 


\section{Características del entorno escolar}

Para el conjunto de variables que corresponden al entorno escolar, se encontró que el puntaje promedio en el área de matemáticas tiene un efecto positivo sobre el desempeño individual. De igual manera el índice socio económico es significativo pero negativo y muy pequeño. Si bien se puede argumentar que puede haber una correlación entre estas dos variables, es claro que el índice socieconómico es una variable discreta que por construcción debería tener una correlación menor que la variable ingreso.

En la Tabla 5, donde sólo se presentan los efectos fijos, tenemos que en promedio, los colegios femeninos tienen una mayor correlación con un buen desempeño promedio en el área de matemáticas y su mejor desempeño a nivel de Bogotá y Nacional está asociado a las competencias comunicativa y de resolución de problemas. Esto muestra que aunque en promedio las niñas tienen un desempeño menor, como grupo son más disciplinadas y en conjunto su desempeño es mejor que el de los colegios sólo de niños. Es importante resaltar, que en promedio, el desempeño de los estudiantes no es significativo en las competencia argumentativa independiente del género del plantel y este resultado se mantiene a nivel nacional y para Bogotá. De igual manera, en lenguaje a las niñas en promedio les va mejor que los niños y se esperaría que esto tuviera un impacto en los resultados en la prueba Saber 11 para el área de matemáticas. Se abre como un tema de investigación para ser explorado en mayor profundidad.

Finalmente, el índice agregado de calidad es sólo significativo para la competencia comunicativa para Bogotá y a nivel nacional.

La Tabla 5 se presenta los resultados para los efectos fijos y los efectos aleatorios. Con respecto a las características del individuo, el modelo captura los efectos fijos ya presentados en la sección anterior. Para dicho conjunto de variables el valor de coeficiente, el signo y el nivel de significancia de los coeficientes se mantiene.

Con respecto a las características del capital social los resultados son robustos en su conjunto frente al modelo de efectos fijos. En efecto, al analizar los resultados de las variables de capital social, los resultados NO son significativos para los niveles educativos del padre y la madre. Además, para aquellos donde se encuentra algún grado de significación estadística, el impacto en general de cada una de las variables para Bogotá y a Nivel Nacional es mínimo (los coeficientes son cercanos a cero).

De igual manera, los resultados con respecto a la Jornada escolar a la cual se asiste, se tiene que para todas las compentencias en Bogotá, las jornadas completa, mañana son significativas y tienen un impacto positivo sobre el desempeño y su rendimiento en promedio es mejor que con respecto a Jornada de la Tarde, mientras que la jornada Noche claramente no es significativo. A nivel nacional, al no existir una gran heterogeneidad en lo referente a la calidad entre planteles oficiales y públicos, allí para todas las jornadas la relación es positiva y significativa, aunque en términos de escala, hay una relación decreciente en el tamaño del coeficiente asociada claramente a la calidad. Este resultado debe conducir a un estudio más riguroso sobre el rendimiento global de los colegios en las Jornadas Tarde y Noche, ya que el logro de una mayor cobertura educativa a través del uso intensivo de la infraestructura educativa parece tener un efecto negativo sobre la calidad y sobre el desempeño al menos en las competencias en matemáticas evaluadas a través de las pruebas Saber 11. 
Es importante señalar, que la no significancia de variables como el tener o no computador, o el del índice socieconómico, pierden significancia para el caso de Bogotá, debido a que dicho efecto es capturado a través de las variables que en el segundo nivel de la estimación capturan las interacciones. En efecto, Lenguaje:Computador es significativa a Nivel nacional, mientras que la interacción Conputador:Índice Socieconómico es negativa y significativa para Bogotá y para el nivel nacional. Finalmente, hay una correlación positiva en la interacción entre el promedio de los resultados de Lenguaje:Matemáticas por plantel, esta interacción es significativa.

De igual manera, el efecto fijo del puntaje promedio en matemáticas se captura a través de los efectos fijos por la interacción con el puntaje promedio obtenido en la prueba de lenguaje. Esta interacción capturada por el modelo total es significativa y explica la varianza de al menos del $25 \%$ para todas las competencias en el caso de Bogotá.

Tabla 6.

HGLM. Efectos Totales (Análisis de Varianza)

\begin{tabular}{|c|c|c|c|c|c|c|}
\hline \multirow[b]{2}{*}{ Variables } & \multicolumn{3}{|c|}{ Bogotá D.C. } & \multirow[b]{2}{*}{ Comunicativa } & \multicolumn{2}{|c|}{ Total Nacional } \\
\hline & Comunicativa & Argumentativa & $\begin{array}{l}\text { Soluc. } \\
\text { Problemas }\end{array}$ & & Argumentativa & $\begin{array}{l}\text { Soluc. } \\
\text { Problemas }\end{array}$ \\
\hline ICC & $55,6 \%$ & $37,2 \%$ & $37,8 \%$ & $45,4 \%$ & $11,4 \%$ & $8,1 \%$ \\
\hline $\begin{array}{l}\text { \% Varianza } \\
\text { explicado dentro de } \\
\text { grupos (within-group) }\end{array}$ & $33,1 \%$ & $38,1 \%$ & $-37,0 \%$ & $5,1 \%$ & $85,4 \%$ & $81,9 \%$ \\
\hline $\begin{array}{l}\text { \% Varianza } \\
\text { explicado entre grupos } \\
\text { (between group) }\end{array}$ & $0,1 \%$ & $-0,1 \%$ & $-0,1 \%$ & $0,5 \%$ & $1,0 \%$ & $0,3 \%$ \\
\hline $\begin{array}{l}\text { Puntaje Saber11 } \\
\text { en Lenguaje }\end{array}$ & $-102,3 \%$ & $63,0 \%$ & $-21,8 \%$ & $-3151,3 \%$ & $97,0 \%$ & $27,9 \%$ \\
\hline Nivel Educativo Madre & $-51,69 \%$ & $-108,01 \%$ & $-116,30 \%$ & $-120832,35 \%$ & $97,02 \%$ & $-948,51 \%$ \\
\hline $\begin{array}{l}\text { Tiene Computador } \\
(\mathrm{Si}=1)\end{array}$ & $40,5 \%$ & $53,2 \%$ & $60,1 \%$ & $-1063,5 \%$ & $87,7 \%$ & $44,2 \%$ \\
\hline $\begin{array}{l}\text { Puntaje promedio } \\
\text { Colegio Matemáticas }\end{array}$ & $5,2 \%$ & $15,2 \%$ & $-29,6 \%$ & $-928,3 \%$ & $53,1 \%$ & $68,4 \%$ \\
\hline $\begin{array}{l}\text { d_colgenero1 } \\
(\text { Femenino=1) }\end{array}$ & $8,3 \%$ & $18,6 \%$ & $-4,7 \%$ & $-1057,1 \%$ & $-94,3 \%$ & $-113,3 \%$ \\
\hline $\begin{array}{l}\text { d_colgenero2 } \\
(\text { Masculino=1) }\end{array}$ & $15,8 \%$ & $-47,8 \%$ & $-32,1 \%$ & $-1385,9 \%$ & $-93,7 \%$ & $-973,1 \%$ \\
\hline $\begin{array}{l}\text { No. Observaciones } \\
\text { No. Grupos }\end{array}$ & $\begin{array}{l}85099 \\
13\end{array}$ & $\begin{array}{l}85099 \\
13\end{array}$ & $\begin{array}{l}85099 \\
13\end{array}$ & $\begin{array}{l}414550 \\
14\end{array}$ & $\begin{array}{l}414550 \\
14\end{array}$ & $\begin{array}{l}414550 \\
14\end{array}$ \\
\hline
\end{tabular}

Fuente: ICFES, Cálculos del autor. 


\section{CONCLUSIONES}

El presente estudio se inscribe en a línea de trabajos cuantitativos que busca medir el desempeño académico de los estudiantes en las pruebas Saber 11 para el caso de Bogotá a través de la estimación de modelos jerárquicos lineales generalizados (Hierarchical Generilized Linear Models-HGLM). La estimación del modelo multinivel de acuerdo con las características de calidad definidas por el Icfes y la naturaleza del plantel (Oficial o No-Oficial) plantea la necesidad de profundizar de manera más rigurosa en varios aspectos.

El bajo desempeño que presentan los estudiantes en la prueba de matemáticas para las tres competencias, donde muy pocos estudiantes tienen logran responder el $50 \%$ de las preguntas en cada competencia, siendo la de resolución de problemas donde el desempeño es más bajo muestra la necesidad de un análisis más detallado de los tipos de representación y los cambios de representación (tratamiento y conversión) que permiten el aprendizaje de los objetos matemáticos. Este análisis implica tener acceso a los enunciados de las preguntas o el poder hacer una prueba piloto con una muestra representativa que busque analizar los resultados con miras a proponer soluciones concretas para mejorar el desempeño en matemáticas.

La correlación positiva entre el resultado en el área de lenguaje y los resultados promedio en el desempeño del área de matemáticas, unido a que a las niñas las niñas en promedio obtienen un mejor desempeño en lenguaje que en matemáticas con relación a los niños, plantea un estudio detallado sobre los procesos de aprendizaje por género tanto en el área de matemáticas como en el área de lenguaje.

Si bien el nivel educativo de los padres, así como el nivel de ocupación del padre no fue significativo, diversos estudios muestran que el nivel educativo del padre que pasa más tiempo con el niño tiene un impacto positivo en el aprendizaje y por ende en el desempeño en las distintas pruebas estandarizadas. Este factor debería incluirse de manera más precisa para mirar su impacto.

El tener computador en la casa muestra un impacto positivo sobre el desempeño y una correlación alta entre los efectos aleatorios al interior de los grupos de estudio definidos en el modelo multinivel. Debido a que esta variable buscaba ser un proxy del accesos a una biblioteca, se debería incluir en próximos estudios pero combinada con el acceso o no a internet.

La Jornada Escolar, como un factor asociado a las características del ambiente que propician la acumulación del capital social muestra un efecto positivo para la Jornada Completa y la Jornada de la Mañana, con relación a la Jornada Tarde. El hecho que la Jornada Nocturna sea positivo y no significativo, muestra que el efecto es igual o peor para la Jornada Tarde que se tiene como referencia. Este resultado muestra las diferencias en el desempeño de acuerdo a las Jornadas y amerita un estudio más riguroso que busque mostrar si el uso intensivo de la infraestructura educativa con miras a aumentar al cobertura tiene un efecto negativo sobre la calidad. 


\section{REFERENCIAS BIBLIOGRÁFICAS}

Acevedo, M. (2007). Fundamentación conceptual área de matemáticas. Grupo de Evaluación de la Educación Superior - ICFES.

Artigue, M. (1990). "Epistémologie et didactique". En: Recherches en Didactique des Mathématiques 10.2-3, págs. 241-286.

Barrera, F. (2005). Impact of Private Provision of Public Education: Empirical Evidence from Bogotá's Concessions Schools. Conference: "Mobilizing the Private Sector for Public Education" Co-sponsored by the World Bank Kennedy School of Government, Harvard University, October 5-6, 2005.

Barrientos, J. y A. Gaviria (2001a). Calidad de la educación y rendimiento académico en Bogotá. Coyuntura Social, 24, 111-127.

Barrientos, J. y A. Gaviria (2001b). Características del plantel y calidad de la educación en Bogotá. Coyuntura Social, 25, 82-98.

Barrientos, J. y A. Gaviria (2001c). Determinantes de la calidad de la Educación en Colombia. Archivos de Economía. Departamento Nacional de Planeación.

Becerra, L., J. González y A. Sarmiento (2000). La incidencia del plantel en el logro educativo del alumno y su relación con el nivel socioeconómico. Coyuntura Social, 22, 53-64.

Bertalanffy, L. (1968). Teoría general de los sistemas: fundamentos, desarrollo, aplicaciones. Fondo de Cultura Económica.

Bliesse, P. (2003). Multilevel Modeling in R(2.3). Working Paper.

Bosh, M., C. Fonseca y J. Gascón (2004). "Incompletitud de las organizaciones matemáticas locales en las instituciones escolares." En: Recherches en Didactique des Mathématiques 24.2-3, págs. 205-250.

Bronfenbrenner, U. (1979). La ecología del desarrollo humano: experimentos en entornos naturales. Ediciones Paidós Iberica, S.A. Barcelona.

Brousseau, G. (1986). Fondements et méthodes de la didactiques des mathématiques. Recherches en Didactique des Mathématiques 7.2, 33-115.

Brousseau, G. (1998). La théorie des situations didactiques. La Pensée Sauvage. Grenoble.

Brown, S., L. Flick y K. Williamson (2005). Social Capital in Engineering Education. Proceedings of the 35th ASEE/IEEE Frontiers in Education Conference, Indianapolis.

Bryk, A y Stephen W. Raudenbush (2002). Hierarchical Linear Models. Applications and Data Analysis Methods. Sage Publications.

Chevallard, Y. (1999). L'analyse des pratiques enseignantes en théorie anthropologique du didactique. Recherches en Didactique des Mathématiques 19.2, 221-266.

Correa, J. (2004). Determinantes del rendimiento educativo de los estudiantes de secundaria en Cali: un análisis multinivel. Revista Sociedad y Economía 6, 81-105. 
Daily, S., W. Eugene y A. Prewitt (2007). The Development of Social Capital in Engi- neering Education to Improve Student Retention. ASEE Southeast Section Conference.

D’Amore, B. (2006). Didáctica de la Matemática. Cooperativa Editorial Magisterio, Bogotá.

Douady, R. (1986). Jeux de cadres et dialectique des mathématiques? Recherches en Didactique des Mathématiques 349-382.

Duval, R. (2004). Semiosis y pensamiento humano. Instituto de Educación y Pedagogía. Universidad del Valle.

Ferrante, F. y F Sabatini (2007). Education, social capital and entrepreneurial selection in Italy. Online at http://mpra.ub.uni-muenchen.de/2451/ MPRA Paper No. 2451, posted 07.

Godino, J. (2002). Un enfoque ontológico y semiótico de la cognición matemática. Recherches en Didactique des Mathématiques 22.2-3, 237-284.

Godino, J. y C. Batanero (1994). Significado institucional y personal de los objetos matemáticos. Recherches en Didactique des Mathématiques 14.3, 325-355.

Godino, J., C. Batanero y R. Roa (2005). An onto-semiotic analysis of combinatorial problems and the solving process by university students. Educational Studies in Mathematics 60.1, 3-36.

Godino, J., A. Contreras y V. Font (2006). Análisis de los procesos de instrucción basado en el enfoque ontológico-semiótico de la cognición matemática. Recherches en Didactique des Mathématiques 26.1.

Godino, J. y col. (2006). Una visión de la Didáctica Francesa desde el enfoque onto-semiótico de la cognición e instrucción matemática. Revista Latinoamericana de Investigación en Matemática Educativa 9.1,117-150.

Hendry, D. (1995). Dynamic Econometrics. Oxford University Press.

Lerouge, A. (2000). La notion de cadre de rationalité. A propos de la droite au collège. Recherches en Didactique des Mathématiques.

Lilienfeld, R. (1984). Teoría de sistemas. Orígenes y aplicaciones en ciencias sociales. Editorial Trillas. México.

Luhmann, N. (1998). Sistemas sociales. Lineamientos para una teoría general. Anthropos Universidad Iberoamericana - CEJA Pontificia Universidad Javeriana.

McGowen, R. (2009). The impact of school facilities on student achievement, attendance, behavior, completion rate and teacher turnover rate in selected Texas high schools. Doctoral dissertation, Texas AM University.

Olsson, M. y G. Sjöstedt, eds. (2005). Systems Approaches and Their Application. Examples from Sweden. Kluwer Academic Publishers. New York.

Putnam (1994). Tuning In, Tuning Out: The Strange Disappearance of Social Capital in America. PS Online: American Political Science Association's Journal of the Profession. 
Rodríguez, J. (2011). Peer Effects and School Resources in Colombia: A Multilevel Analysis of Performance on the 2008 College Entrance Exam. Stanford University: School of Education.

Schuller, T. (2000). The complementary roles of human and social capital. Birkbeck College, University of London.

Stanton-Salazar, R. y S. Dornbush (1995). Social Capital and the Reproduction of Inequality: Information Networks among Mexican-origin High School Students. Sociology of Education 68, 116-135.

Ulrich, W. (2001). The Quest for Competence in Systemic Research and Practice. Systems Research and Behavioral Science 18.1, 3-28.

Vasco, C. (1995). "La teoría general de procesos y sistemas". En: Misión, Ciencia, Educación y Desarrollo. Educación para el Desarrollo.

Vergnaud, G. (1990). La théorie des champs conceptuels. Recherches en Didactique des Mathématiques 10.2-3, 133-170.

Woolcock, M. (2000). The place of social capital in understanding social and economic outcomes. Working Paper, World Bank. 